\title{
Dielectric Relaxation of Mixtures of $N$-Methylacetamide and $N, N$-Dimethylformamide Solved in Benzene Using Microwave Absorption Data
}

\author{
Raman Kumar, V. S. Rangra, D. R. Sharma, N. Thakur, and N. S. Negi \\ Electronics Research Laboratory, Physics Department, Himachal Pradesh University, Shimla 171005, \\ India \\ Reprint requests to Prof. V.S. R.; E-mail: vs_rangra@yahoo.co.in
}

Z. Naturforsch. 61a, 197 - 204 (2006); received November 24, 2005

The dielectric relaxation times $\tau$ and dipole moments $\mu$ of $N$-methylacetamide (NMA) mixed with $N, N$-dimethylformamide (DMF) in benzene solutions have been obtained using standard standing wave microwave techniques and Gopala Krishna's single frequency $(9.90 \mathrm{GHz})$ concentration variational method at $25,30,35$, and $40^{\circ} \mathrm{C}$. The energy parameters $\left(\Delta H_{\varepsilon}, \Delta F_{\varepsilon}, \Delta S_{\varepsilon}\right)$ for the dielectric relaxation process of mixtures with equal amounts of NMA and DMF have been calculated and compared with the corresponding energy parameters $\left(\Delta H_{\eta}, \Delta F_{\eta}, \Delta S_{\eta}\right)$ for the viscous flow. On the basis of the observations it is found that the dielectric relaxation process can be treated as a rate process like the viscous flow. Solute-solute and solute-solvent types of the molecular associations have been predicted.

Key words: Dielectric Relaxation; Solute-Solute Interaction; Relaxation Times;

Microwave Absorption Studies.

\section{Introduction}

The dielectric relaxation of binary mixtures of polar molecules in non-polar solvents has often been studied by microwave absorption methods [1-7]. Amides form the basic building blocks of proteins; therefore dielectric relaxation studies of their mixtures are of great interest. $N$-Methylacetamide (NMA) is a dipolar, aprotic solvent with the dipole moment $\mu=3.50 \mathrm{D}$ and the dielectric constant $\varepsilon^{\prime}=178.9$ at $30{ }^{\circ} \mathrm{C}$ and 165.5 at $40{ }^{\circ} \mathrm{C}$ [8]. $N, N$-Dimethylformamide (DMF) is a non-aqueous aprotic solvent with the dipole moment $\mu=3.86 \mathrm{D}$ and the dielectric constant $\varepsilon^{\prime}=36.71$ [8]. DMF is a colourless liquid, in the range -60 to $153{ }^{\circ} \mathrm{C}$, has a low vapour pressure and good solubility for many substances [9]. It is possible to prepare binary mixtures of a dielectric constant lying between that of NMA and DMF. This motivated us to undertake experimental work concerned with the dielectric relaxation in binary mixtures of NMA and DMF.

Dielectric relaxation studies in the microwave region provide information about self-association, solute-solute and solute-solvent type associations among the polar molecules [10-12]. The present study deals with the molecular association in mixture of
NMA and DMF at $25,30,35$ and $40{ }^{\circ} \mathrm{C}$. Standard standing wave microwave techniques and the single frequency concentration variational method of Gopala Krishna have been used. This method makes use of benzene as the suspension medium for the study of the suspended rotating molecules. It consists in measuring dielectric constant $\left(\varepsilon^{\prime}\right)$ and the dielectric loss $\left(\varepsilon^{\prime \prime}\right)$ of series of dilute solutions of polar molecules benzene.

\section{Experimental}

NMA (GC grade) from Fluka, Germany was purified by three-fold crystallization. DMF (Sisco Research Laboratories, Bombay) was dried on $4 \AA$ molecular sieves for $48 \mathrm{~h}$ with occasional shakings and then distilled through a long vertical fractionating column. The middle fractions were collected for use. Benzene (Central Drug House Pvt Ltd, New Delhi) was dried by refluxing over freshly cut sodium metal for $6-8 \mathrm{~h}$ and then distilled through a long vertical fractionating column. The middle fraction of the distilled benzene was used. An X-band microwave bench was used to measure the wavelength in the dielectric medium and the voltage standing wave ratio (VSWR) using a shortcircuiting plunger. The set up was tuned at a microwave 
frequency of $9.90 \mathrm{GHz}$. The experimental techniques of Arrawatia et al., used by Sharma and Sharma [13] for microwave measurements, were used. Sets of dilute solutions of the binary mixtures in the benzene solutions were prepared. All measurements were carried out at $25,30,35$ and $40{ }^{\circ} \mathrm{C}$ by circulating water around the dielectric cell through a thermostat (Dr. R. Wobser GmbH \& Co., Lauda Germany). The precision and working of the equipment was tested by measuring the dipole moments of purified acetone, methanol and pyridine. The viscosity and density of the solutions were measured by an Ubbelohde viscometer and a sealable pycnometer, respectively.

Using standard standing wave microwave techniques and following the method of Heston et al. [14], the dielectric constant $\left(\varepsilon^{\prime}\right)$ and the dielectric loss $\left(\varepsilon^{\prime \prime}\right)$ of the dilute solutions of NMA, DMF and (NMA+DMF) binary mixtures containing 30, 50 and $70 \mathrm{~mol} \% \mathrm{DMF}$ in benzene at $25{ }^{\circ} \mathrm{C}$ have been calculated and given in Table 1.

The following equations have been used:

$$
\varepsilon^{\prime}=\left(\frac{\lambda_{\mathrm{o}}}{\lambda_{\mathrm{c}}}\right)^{2}+\left(\frac{\lambda_{\mathrm{o}}}{\lambda_{\mathrm{d}}}\right)^{2}
$$

and

$$
\varepsilon^{\prime \prime}=\frac{2}{\pi}\left(\frac{\lambda_{\mathrm{o}}}{\lambda_{\mathrm{d}}}\right)^{2} \cdot \frac{\lambda_{\mathrm{g}}}{\lambda_{\mathrm{d}}}\left(\frac{\mathrm{d} \rho}{\mathrm{d} n}\right),
$$

where $\lambda_{\mathrm{o}}, \lambda_{\mathrm{c}}, \lambda_{\mathrm{g}}$ and $\lambda_{\mathrm{d}}$ are the wavelengths in free space, the cut-off wavelength, the waveguide wavelength and the wavelength in the waveguide filled with solution, respectively. $\rho$ is the inverse of the voltage standing wave ratio (VSWR) and $\mathrm{d} \rho / \mathrm{d} n$ is the slope of the curve of $\rho$ versus $n$, where $n$ is the integer $(1,2,3,4, \ldots)$ such that $\left(n \lambda_{\mathrm{d}} / 2\right)$ represents the length of the dielectric filled waveguide. $\varepsilon^{\prime}$ and $\varepsilon^{\prime \prime}$ were reproducible within $\pm 0.5 \%$ and $\pm 1.67 \%$, respectively. Following Gopala Krishna's single frequency concentration variational method [15], the dielectric relaxation time $(\tau)$ and the dipole moment $(\mu)$ have been calculated.

Gopala Krishna's method makes use of Debye's theory of dielectric relaxation. Debye's equation for the complex dielectric constant of the dielectric medium as a function of the frequency of the applied electric field [16] can be written as

$$
\frac{\varepsilon^{*}-1}{\varepsilon^{*}+2}=\frac{\varepsilon_{\infty}-1}{\varepsilon_{\infty}+2}+\frac{4 \pi N_{1} \mu^{2}}{9 k T} \frac{1}{1+j \omega \tau},
$$

where $N_{1}$ is the number of polar molecules per unit volume and $\varepsilon^{*}=\varepsilon^{\prime}-j \varepsilon^{\prime \prime}$ is the complex dielectric constant of the medium. $\varepsilon_{\infty}$ is the optical permittivity, and $\omega$ the angular frequency.

Separating real and imaginary parts of both sides of (3) yields

$$
\begin{aligned}
& \frac{\varepsilon^{\prime 2}+\varepsilon^{\prime}+\varepsilon^{\prime \prime 2}-2}{\left(\varepsilon^{\prime}+2\right)^{2}+\varepsilon^{\prime \prime 2}}=\frac{\varepsilon_{\infty}-1}{\varepsilon_{\infty}+2}+\frac{4 \pi N_{1} \mu^{2}}{9 k T} \frac{1}{1+\omega^{2} \tau^{2}}, \\
& \frac{3 \varepsilon^{\prime \prime}}{\left(\varepsilon^{\prime}+2\right)^{2}+\varepsilon^{\prime \prime 2}}=\frac{4 \pi N_{1} \mu^{2}}{9 k T} \frac{\omega \tau}{1+\omega^{2} \tau^{2}} .
\end{aligned}
$$

Putting

$$
\begin{aligned}
& X=\frac{\varepsilon^{\prime 2}+\varepsilon^{\prime}+\varepsilon^{\prime \prime 2}-2}{\left(\varepsilon^{\prime}+2\right)^{2}+\varepsilon^{\prime \prime 2}}, \\
& Y=\frac{3 \varepsilon^{\prime \prime}}{\left(\varepsilon^{\prime}+2\right)^{2}+\varepsilon^{\prime \prime 2}},
\end{aligned}
$$

and

$$
P=\frac{\varepsilon_{\infty}-1}{\varepsilon_{\infty}+2}
$$

in the above equations, one gets

$$
X=P+\frac{Y}{\omega \tau} .
$$

The value of $P$ in (6) slightly varies in the range of concentrations of dilute solutions. But as the variation of $\varepsilon^{\prime}$ and $\varepsilon^{\prime \prime}$ is by far higher than the variation of $P$ due to the change in concentration of dilute solutions at microwave frequencies, it could be treated as constant. From the slope of the curve $Y$ versus $X$ the relaxation time $\tau$ of polar molecules in non-polar solvents could be determined. It can be written as

$$
\tau=\frac{\lambda_{\mathrm{o}}}{2 \pi c}\left(\frac{\mathrm{d} Y}{\mathrm{~d} X}\right) .
$$

For the determination of dipole moment $\mu$ (6) can be written as

$$
X=P+K w d_{12}
$$

with

$$
K=\frac{4 \pi N \mu^{2}}{9 k T M\left(1+\omega^{2} \tau^{2}\right)}
$$

and

$$
N_{1}=\frac{N d_{12} w}{M},
$$


Table 1. Values of the dielectric constant $\left(\varepsilon^{\prime}\right)$, dielectric loss $\left(\varepsilon^{\prime \prime}\right)$, relaxation time $(\tau)$ and dipole moment $(\mu)$ of NMA, DMF, and (NMA+DMF) mixtures in benzene at $25^{\circ} \mathrm{C}$.

\begin{tabular}{|c|c|c|c|c|c|}
\hline $\begin{array}{l}\text { Mole fraction } \\
\text { of DMF in } \\
\text { (NMA+DMF) } \\
\text { mixtures }\end{array}$ & $\begin{array}{l}\text { Weight } \\
\text { fraction of } \\
\text { solute in } \\
\text { benzene }\end{array}$ & $\begin{array}{c}\mathcal{E}^{\prime} \\
( \pm 0.5 \%)\end{array}$ & $\begin{array}{c}\varepsilon^{\prime \prime} \\
{\left[10^{-12} \mathrm{~s}\right]} \\
( \pm 1.67 \%)\end{array}$ & $\tau$ & $\mu$ [D] \\
\hline \multirow[t]{5}{*}{$\overline{0.0}$} & .0049 & 2.355 & 0.0418 & 4.13 & $3.74\left(\mu_{\mathrm{NMA}}\right)$ \\
\hline & .0058 & 2.370 & 0.0468 & & \\
\hline & .00736 & 2.402 & 0.0558 & & \\
\hline & .00856 & 2.432 & 0.0632 & & \\
\hline & .0093 & 2.443 & 0.0672 & & \\
\hline \multirow[t]{5}{*}{0.30} & .00433 & 2.362 & 0.0243 & 4.68 & \\
\hline & .00533 & 2.388 & 0.0323 & & \\
\hline & .00673 & 2.417 & 0.0418 & & \\
\hline & .0080 & 2.450 & 0.0536 & & \\
\hline & .00897 & 2.470 & 0.0578 & & \\
\hline \multirow[t]{5}{*}{0.50} & .00406 & 2.348 & 0.0240 & 5.03 & \\
\hline & .00523 & 2.366 & 0.0304 & & \\
\hline & .00667 & 2.391 & 0.0396 & & \\
\hline & .00813 & 2.424 & 0.0499 & & \\
\hline & .00903 & 2.435 & 0.0534 & & \\
\hline \multirow[t]{5}{*}{0.70} & .0032 & 2.334 & 0.0185 & 4.29 & \\
\hline & .00452 & 2.359 & 0.0253 & & \\
\hline & .00727 & 2.417 & 0.0418 & & \\
\hline & .00862 & 2.450 & 0.0517 & & \\
\hline & .0103 & 2.493 & 0.0627 & & \\
\hline \multirow[t]{5}{*}{1.00} & .0027 & 2.310 & 0.0153 & 4.13 & $3.84\left(\mu_{\mathrm{DMF}}\right)$ \\
\hline & .00476 & 2.352 & 0.0268 & & \\
\hline & .00586 & 2.380 & 0.0347 & & \\
\hline & .00855 & 2.435 & 0.0490 & & \\
\hline & .00981 & 2.466 & 0.0580 & & \\
\hline
\end{tabular}

where $N$ is the Avogadro number, $M$ the molecular weight of the polar substance, $w$ the weight fraction and $d_{12}$ the density of the solution. At low concentrations, the variation of the density of the solution with the weight fraction $w$ may be taken as linear

$$
d_{12}=d_{0}(1+\alpha w)
$$

where $d_{0}$ is the density of the solvent. In the limited experimental range of concentration variation, the graph between $X$ and $w$ can be taken as a straight line with its slope $(\mathrm{d} X / \mathrm{d} w)$ as $K d_{0}$. From this the value of the dipole moment may be calculated, using the relation

$$
\mu^{2}=\frac{9 k T M}{4 \pi N d_{0}}\left[1+\left(\frac{\mathrm{d} Y}{\mathrm{~d} X}\right)^{2}\right] \frac{\mathrm{d} X}{\mathrm{~d} w} .
$$

\section{Results and Discussions}

The values of $\varepsilon^{\prime}, \varepsilon^{\prime \prime}, \tau, \mu$ and $\eta$ thus determined are shown in Tables 1, 2 and 3, respectively. It is found that:
Table 2. Dielectric relaxation time $(\tau)$ and dipole moment $(\mu)$ for different mole fractions of (NMA+DMF) mixtures in benzene at different temperatures $(t)$.

\begin{tabular}{cccc}
\hline$t$ & $\begin{array}{c}\text { Mole fraction of DMF } \\
\text { in binary mixtures }\end{array}$ & $\begin{array}{c}\tau \\
{\left[{ }^{\circ} \mathrm{C}\right]}\end{array}$ & $\mu[\mathrm{D}]$ \\
\hline 25 & 0.00 & 4.13 & $3.74\left(\mu_{\mathrm{NMA}}\right)$ \\
& 0.30 & 4.68 & \\
& 0.50 & 5.03 & \\
& 0.70 & 4.29 & $3.84\left(\mu_{\mathrm{DMF}}\right)$ \\
30 & 1.00 & 4.13 & $3.62\left(\mu_{\mathrm{NMA}}\right)$ \\
& 0.00 & 4.02 & \\
& 0.30 & 4.59 & \\
& 0.50 & 4.92 & \\
35 & 0.70 & 4.23 & \\
& 1.00 & 3.79 & $3.91\left(\mu_{\mathrm{DMF}}\right)$ \\
& 0.00 & 3.91 & $3.55\left(\mu_{\mathrm{NMA}}\right)$ \\
& 0.30 & 4.49 & \\
40 & 0.50 & 4.81 & \\
& 0.70 & 4.17 & \\
& 1.00 & 3.53 & $3.99\left(\mu_{\mathrm{DMF}}\right)$ \\
& 0.00 & 3.80 & $3.52\left(\mu_{\mathrm{NMA}}\right)$ \\
& 0.30 & 4.40 & \\
& 0.50 & 4.70 & \\
& 0.70 & 4.11 & $4.06\left(\mu_{\mathrm{DMF}}\right)$ \\
\hline
\end{tabular}

Table 3. Density $\left(d_{\mathrm{o}}\right)$, molar volume $(V)$ and viscosity $(\eta)$ of benzene at different temperatures $(t)$.

\begin{tabular}{cccc}
\hline $\begin{array}{c}\left.{ }^{\circ} \mathrm{C}\right] \\
{\left[{ }^{\circ}\right]}\end{array}$ & $\begin{array}{c}d_{\mathrm{o}} \\
{\left[\mathrm{g} / \mathrm{cm}^{3}\right]}\end{array}$ & $\begin{array}{c}\eta \\
{\left[\mathrm{P} \cdot 10^{5}\right]}\end{array}$ & $\begin{array}{c}V \\
{\left[\mathrm{M} / d_{\mathrm{o}}\right]}\end{array}$ \\
\hline 25 & 0.8736 & 610 & 89.41 \\
30 & 0.8708 & 567 & 89.70 \\
35 & 0.8630 & 525 & 90.51 \\
40 & 0.8574 & 495 & 91.10 \\
\hline
\end{tabular}

Table 4. Relaxation time $(\tau)$, free energies of activation $\left(\Delta F_{\varepsilon}, \Delta F_{\eta}\right.$ in $\left.\mathrm{kJ} \mathrm{mol}^{-1}\right)$, enthalpies of activation $\left(\Delta H_{\varepsilon}\right.$, $\Delta H_{\eta}$ in $\left.\mathrm{kJ} \mathrm{mol}^{-1}\right)$ and entropies of activation $\left(\Delta S_{\mathcal{\varepsilon}}, \Delta S_{\eta}\right.$ in $\mathrm{J} \mathrm{mol}^{-1} \mathrm{deg}^{-1} \mathrm{~K}^{-1}$ ) for (NMA+DMF) mixtures containing $50 \mathrm{~mol} \% \mathrm{DMF}$ in benzene solutions.

\begin{tabular}{cccccccc}
\hline $\begin{array}{c}t \\
{\left[{ }^{\circ} \mathrm{C}\right]}\end{array}$ & $\begin{array}{c}\left.\tau 10^{-12} \mathrm{~s}\right] \\
( \pm 0.5)\end{array}$ & $\Delta F_{\varepsilon}$ & $\Delta H_{\varepsilon}$ & $\Delta S_{\varepsilon}$ & $\Delta F_{\eta}$ & $\Delta H_{\eta}$ & $\Delta S_{\eta}$ \\
\hline 25 & 5.03 & 8.523 & 0.9284 & -25.485 & 12.180 & 10.969 & -4.064 \\
30 & 4.92 & 8.652 & 0.9284 & -25.490 & 12.209 & 10.969 & -4.092 \\
35 & 4.81 & 8.779 & 0.9284 & -25.489 & 12.236 & 10.969 & -4.114 \\
40 & 4.70 & 8.903 & 0.9284 & -25.478 & 12.299 & 10.969 & -4.249 \\
\hline
\end{tabular}

1. The dielectric constant $\left(\varepsilon^{\prime}\right)$ and dielectric loss $\left(\varepsilon^{\prime \prime}\right)$ vary linearly with the weight fraction of the solute in benzene for all binary mixtures. Figures 1 and 2 show one such variation at $25{ }^{\circ} \mathrm{C}$ and $0.50 \mathrm{~mol} \%$ of DMF in binary mixtures.

2. The experimental curve between $X$ and $Y$ for all concentration ranges of the binary mixtures are linear (Fig. 3).

3. The plot between $X$ and $w$ is also linear (Fig. 4). 

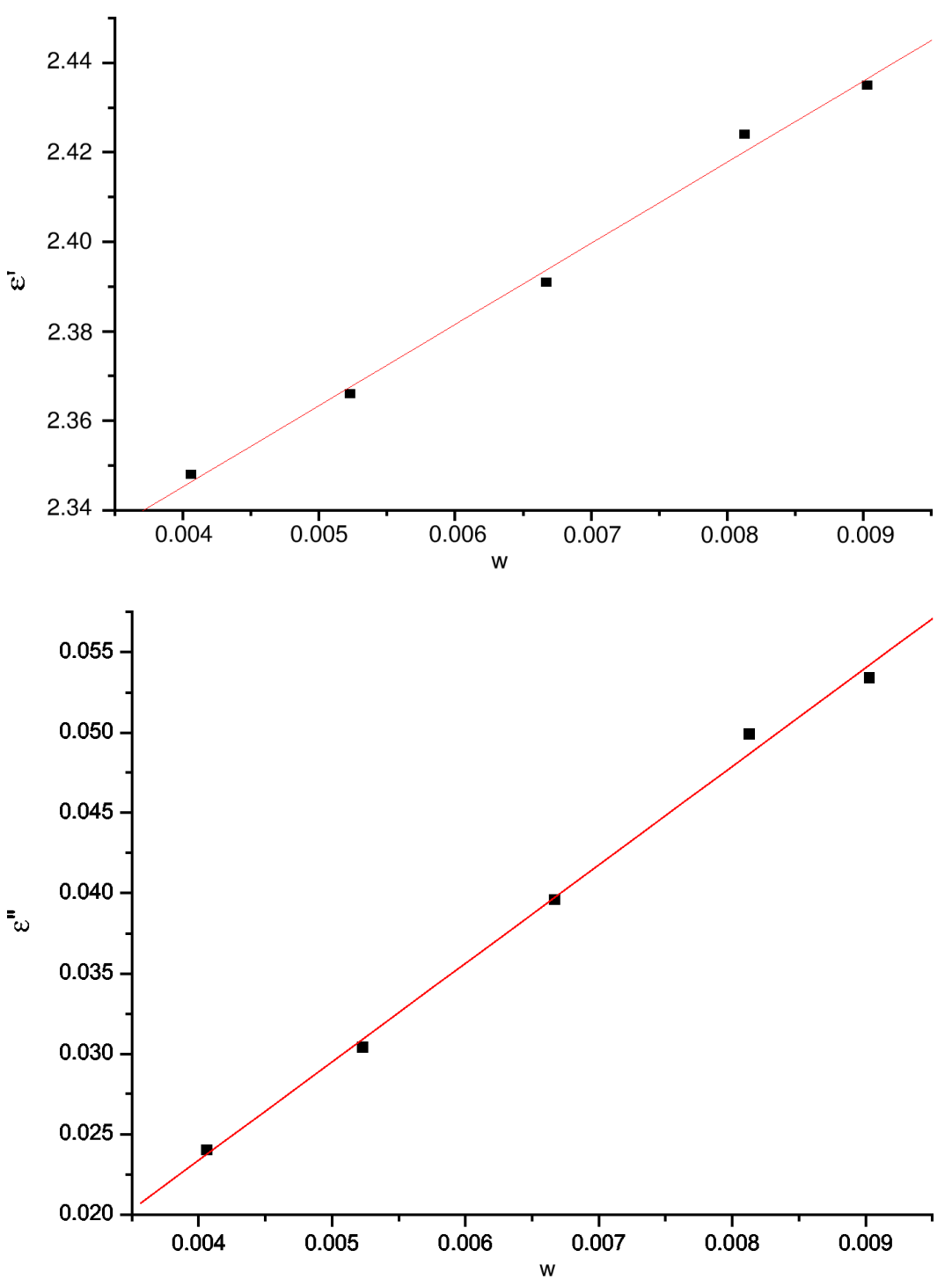

Fig. 1. Variation of the dielectric constant $\left(\varepsilon^{\prime}\right)$ with the weight fraction $(w)$ of the solute at $0.50 \mathrm{~mol} \%$ DMF in (NMA+DMF) binary mixtures at $25^{\circ} \mathrm{C}$.

Fig. 2. Variation of the dielectric loss $\left(\varepsilon^{\prime \prime}\right)$ with the weight fraction $(w)$ of the solute at $0.50 \mathrm{~mol} \% \mathrm{DMF}$ in (NMA+DMF) binary mixtures at $25^{\circ} \mathrm{C}$.

The above experimental results ensure the applicability of Gopala Krishna's method in the studied concentration range of the binary mixtures in the benzene solutions. It is found that the dipole moment of pure NMA and DMF in benzene solution is very close to the literature values of the dipole moment. This shows that pure NMA and pure DMF exist in the monomer form in the benzene solution.

The energy parameters $\left(\Delta H_{\varepsilon}, \Delta F_{\varepsilon}, \Delta S_{\varepsilon}\right)$ for the dielectric relaxation process for the (NMA+DMF) binary mixture containing $50 \mathrm{~mol} \% \mathrm{DMF}$ in benzene at 25 , 30,35 and $40{ }^{\circ} \mathrm{C}$ and the corresponding energy parameters $\left(\Delta H_{\eta}, \Delta F_{\eta}, \Delta S_{\eta}\right)$ for the viscous flow have been calculated (Table 4) by using the Eyring et al. [17] relations for the rate process

$$
\begin{gathered}
\tau=\frac{h}{k T} \exp \left(\frac{\Delta F_{\varepsilon}}{R T}\right), \\
\Delta F_{\varepsilon}=\Delta H_{\varepsilon}-T \Delta S_{\varepsilon}, \\
\eta=\frac{h N}{V} \exp \left(\frac{\Delta F_{\eta}}{R T}\right),
\end{gathered}
$$

and

$$
\Delta F_{\eta}=\Delta H_{\eta}-T \Delta S_{\eta}
$$



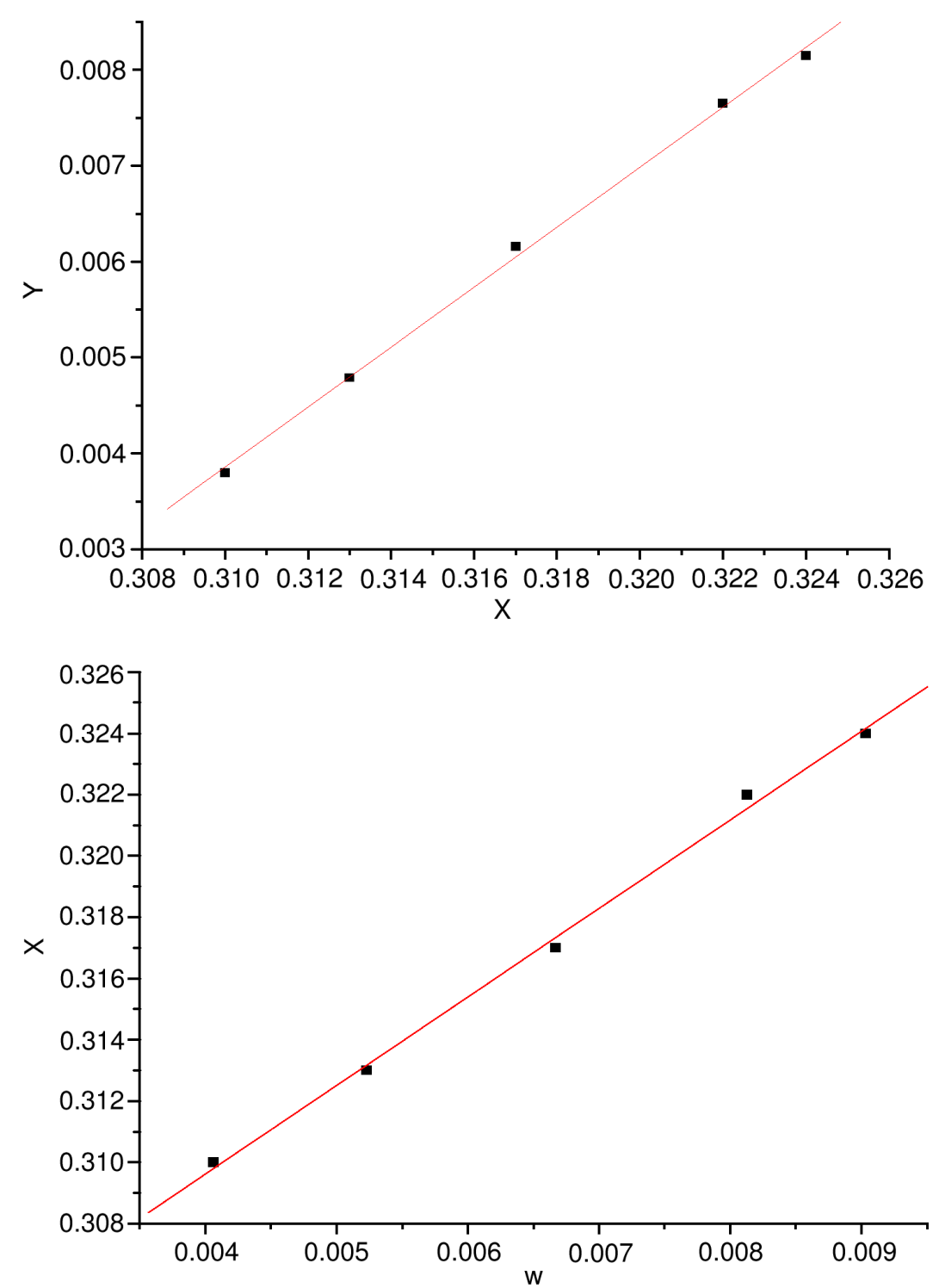

Fig. 3. $Y$ versus $X$ at $0.50 \mathrm{~mol} \%$ DMF in (NMA+DMF) binary mixtures. where $V$ is the molar volume of the solvent. $\Delta H_{\varepsilon}, \Delta F_{\varepsilon}$ and $\Delta S_{\varepsilon}$ are the enthalpy, free energy and entropy of activation, respectively, for the dielectric relaxation process and $\Delta H_{\eta}, \Delta F_{\eta}$ and $\Delta S_{\eta}$ are corresponding parameters for the viscous flow. The plot of $\log (\tau T)$ versus $10^{3} / T$ (Fig. 5) and $\log (\eta)$ versus $10^{3} / T$ (Fig. 6) according to (10) and (12) were found to be linear, which shows that both relaxation and viscous processes can be considered as rate processes. The $\Delta H_{\varepsilon}$ and $\Delta H_{\eta}$ values were computed from the slope of the linear plot of $\log (\tau T)$ and $\log (\eta)$ versus $10^{3} / T$, respectively, using the relation: slope $=\Delta H / 2.303 R$.
Both sets of energy parameters along with the dielectric relaxation time $(\tau)$ for (NMA+DMF) binary mixtures containing $50 \mathrm{~mol} \% \mathrm{DMF}$ in benzene solution at $25,30,35$ and $40{ }^{\circ} \mathrm{C}$ have been summarized in Table 4.

Table 2 shows the dielectric relaxation times $(\tau)$ and dipole moments $(\mu)$ for different mole fractions of (NMA+DMF) binary mixtures at different temperatures in the benzene solution. The relaxation time varies non-linearly with the increase in the mole fraction of DMF in the binary mixtures (NMA+DMF) at all temperatures $\left(25,30,35\right.$ and $\left.40{ }^{\circ} \mathrm{C}\right)$. The relax- 

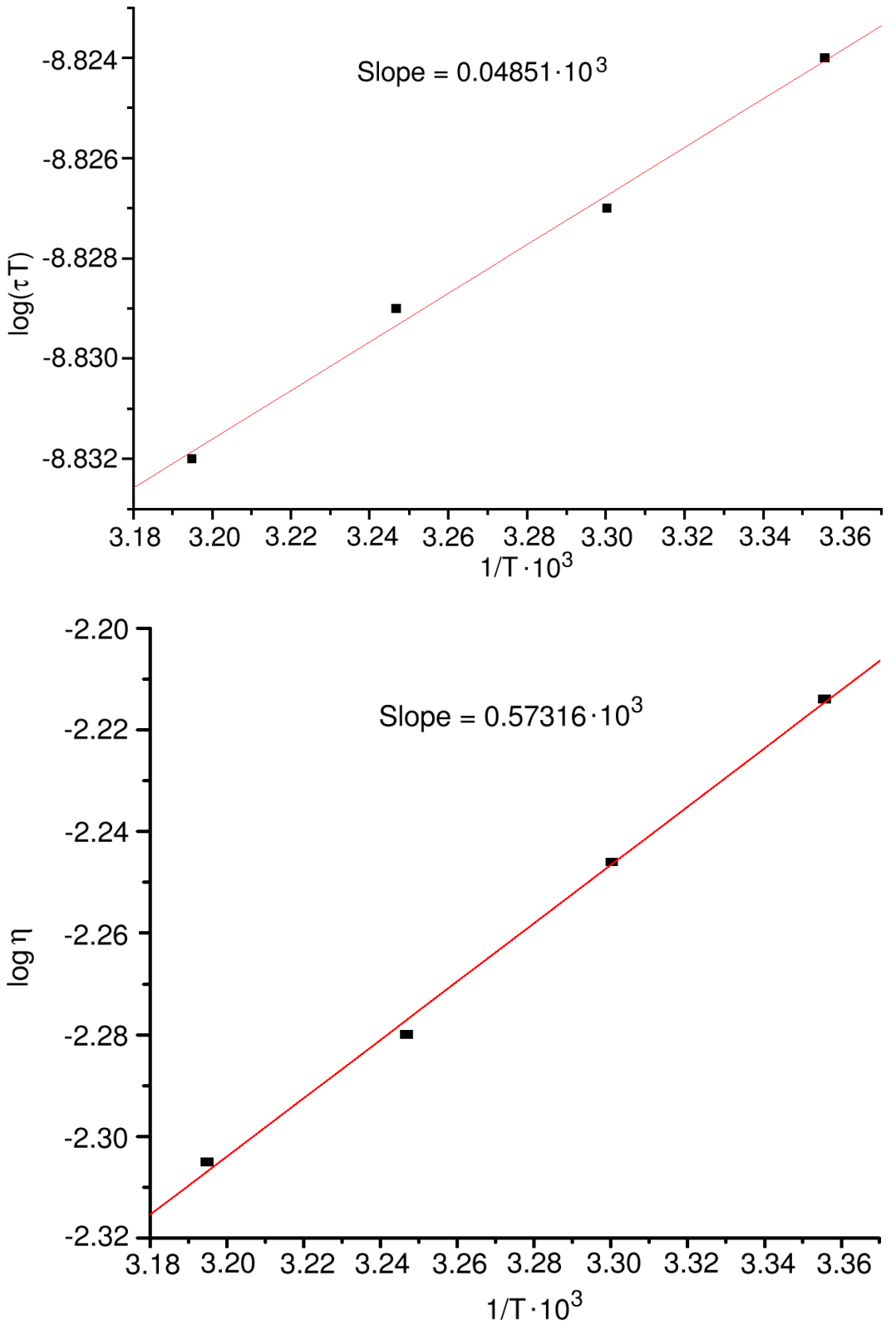

Fig. 5. $\log (\tau T)$ versus $10^{3} / T$ at $0.50 \mathrm{~mol} \%$ DMF in (NMA+DMF) binary mixtures. ation time depends on the size and shape of the rotating molecular entities in the solution. This method determines the average value of the relaxation time for the molecular entities in the solution. The linear variation of the relaxation time from its value corresponding to one constituent to the value corresponding to the other constituent with the mole fraction variation in the whole concentration range may be taken as the absence of any solute-solute association in the mixtures. On the other hand, non-linear variation of the relaxation time with the mole fraction is interpreted as possible solutesolute molecular association in the binary mixtures.

It is interesting to note that the relaxation times of (NMA+DMF) mixtures increases as the mole fraction of DMF in (NMA+DMF) is increased from 0 to 0.5 . After this mole fraction the relaxation time starts decreasing and finally drops to a value of $4.13 \cdot 10^{-12} \mathrm{~s}$ at the mole fraction of DMF equal to 1.00 at $25{ }^{\circ} \mathrm{C}$. 


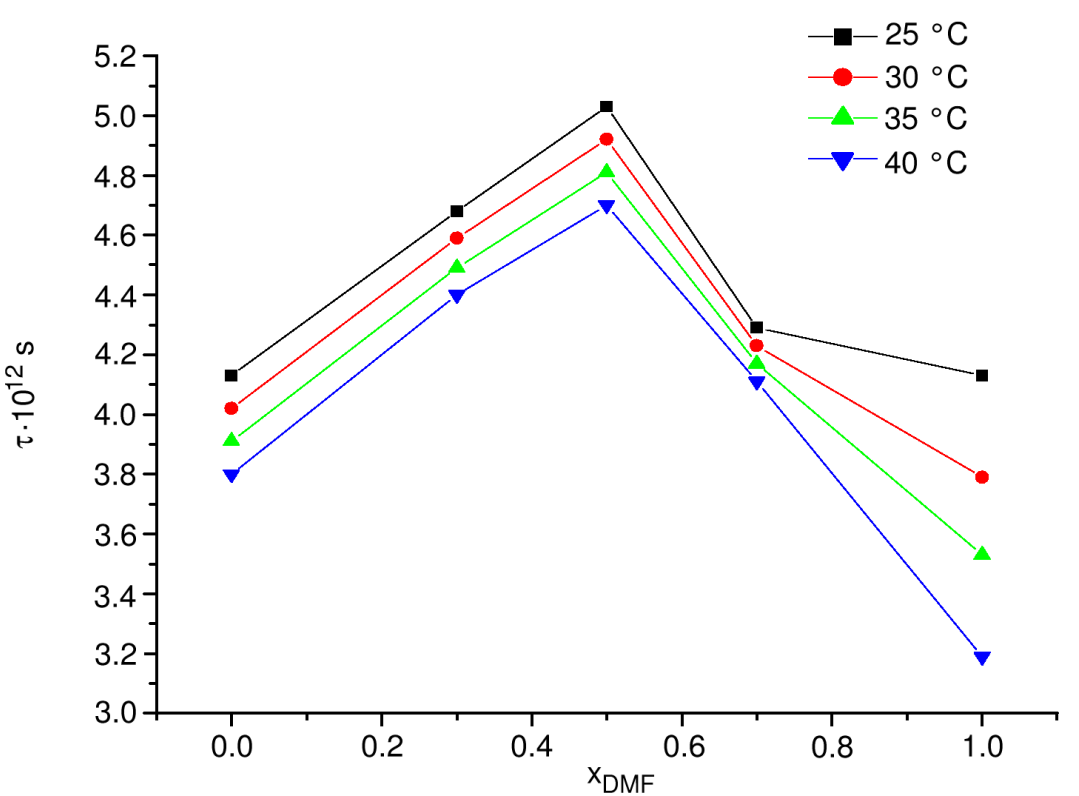

Fig. 7. Relaxation time $(\tau)$ versus mole fraction of DMF $\left(x_{\mathrm{DMF}}\right)$ in (NMA+DMF) mixtures in benzene at different temperatures.
The plot of $\tau$ versus $x_{\mathrm{DMF}}$ is shown in Figure 7. This solute-solute type of molecular association is maximum for equal mole fractions of NMA and DMF. For this content, the relaxation time of the molecular entity becomes longer than that of the individual molecules. The molecular association between NMA and DMF is maximum at the 50:50 mol\% ratio and then decreases at higher mol\% DMF in the mixtures. In its whole concentration range, the relaxation time of $(\mathrm{NMA}+\mathrm{DMF})$ mixtures remains longer than that of pure DMF. Therefore, the solute-solute type of molecular association between NMA and DMF is indicated in its entire concentration range. The solute-solute molecular association arising from the interaction of the fractional negative charge of the oxygen atom of NMA and fractional positive charge of the hydrogen atom of DMF molecules may be proposed (Fig. 8). The dipole moment of NMA varies slightly with rise of temperature and approaches the literature value. The small variation of the dipole moment with rising of temperature may be associated with the breaking nature of the solutesolvent association. The interaction of the fractional positive charge at the C-atom in NMA molecules and the $\pi$-delocalized electron cloud in the benzene ring is shown in the Figure 9.

The observations show that the free energy of activation $\left(\Delta F_{\varepsilon}\right)$ of the dielectric relaxation is smaller than the free energy of activation $\left(\Delta F_{\eta}\right)$ of the viscous flow. This may be explained by the fact that the dielectric<smiles>CN1C(=O)[C@H]2OCCN2C1=O</smiles>

Fig. 8. Solute-solute associations between NMA and DMF.<smiles>CNC(C)([O])c1ccccc1</smiles>

Fig. 9. Solute-solvent association of NMA in benzene.

relaxation involves the rotation of molecular entities, whereas in the viscous flow the rotational as well as the translational motion of the molecules is involved. It is found that the enthalpy of activation for the dielectric relaxation $\left(\Delta H_{\varepsilon}\right)$ is smaller than the enthalpy of activation $\left(\Delta H_{\eta}\right)$ for the viscous flow. The enthalpy of activation depends on the local environment of the molecules. Different values for the enthalpy of activation indicate that the dielectric relaxation and viscous flow involve the breaking of bonds with neighbouring molecules in a different way and to a different extent. The entropy of a system is a measure of the orderly na- 
ture of the system. If the environment of the system is co-operative for the activated process, then the change in entropy $\left(\Delta S_{\varepsilon}\right)$ becomes $-v e$. Whereas the $+v e$ value of the change in the entropy $\left(\Delta S_{\varepsilon}\right)$ for the activation indicates the non-cooperative environment of the sys-

[1] R. Kumar and V. S. Rangra, Z. Phys. Chem. 219, 169 (2005).

[2] V.S. Rangra and D. R. Sharma, Indian J. Pure Appl. Phys. 42, 921 (2004).

[3] S. L. Abd-El-Messieh, J. Mol. Liq. 95, 167 (2002).

[4] A. K. Sharma, D. R. Sharma, K. C. Sharma, and D. S. Gill, Z. Phys. Chem. 141, 15 (1984).

[5] A. K. Sharma, D. R. Sharma, and D. S. Gill, J. Phys. D 18, 1199 (1985)

[6] A. D. Vyas and V. A. Rana, Indian J. Pure Appl. Phys. 40, 69 (2002).

[7] S. K. Sit, N. Ghosh, U. Saha, and S. Acharya, Indian J. Phys. 71B, 553 (1997).

[8] J. J. Lagowski, The Chemistry of Non-Aqueous Solvents, Vol. II, Academic Press, New York and London 1967, p. 195.

[9] A. K. Covington and T. Dickinson, Physical Chemistry of Organic Solvent Systems, Plenum Press, London and New York 1973, p. 14. tem, and the activated state is unstable. In the present case, it is observed that the change in entropy of the dielectric relaxation process is $-v e$, indicating that the environment of the system is co-operative, like that of the activated viscous flow state.

[10] S. S. Krishnamurthy and S. Sundrarajan, J. Phys. Chem. 73, 4036 (1969).

[11] A. K. Sharma and D. R. Sharma, J. Phys. Soc. Jpn. 53, 4471 (1984).

[12] J. S. Dhull, D. R. Sharma, D. S. Gill, and K. N. Lakshminarayana, Indian J. Phys. 56B, 334 (1982).

[13] A. Sharma and D. R. Sharma, J. Phys. Soc. Jpn. 61, 1049 (1992).

[14] W. M. Heston (Jr), A. D. Frankin, E. L. Hennelly, and C. P. Smyth, J. Am. Chem. Soc. 72, 3443 (1950).

[15] K. V. Gopala Krishna, Trans. Faraday Soc. 33, 767 (1957).

[16] N.E. Hill, W.E. Vaughan, A. H. Price, and M. Davies, Dielectric Properties and Molecular Behaviour, Van Nostrand Reinhold Company, London 1969, p. 63.

[17] H. Eyring, S. Glasstone, and K. J. Laidler, Theory of Rate Process, Mc Graw Hill, New York 1941, p. 541. 